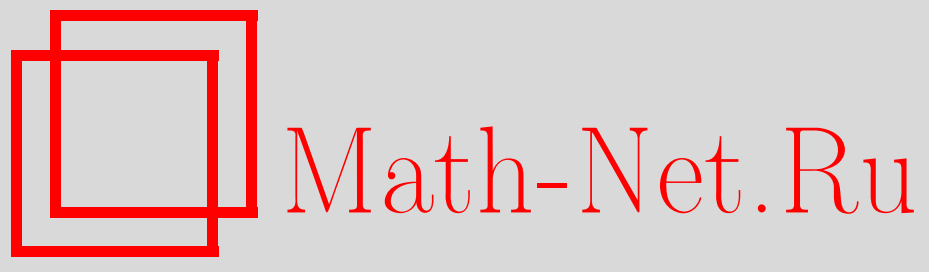

В. А. Дремов, Вычисление двух пар Белого степени 8, УМН, 2009, том 64, выпуск 3, 183-184

DOI: https://doi.org/10.4213/rm9265

Использование Общероссийского математического портала Math-Net.Ru подразумевает, что вы прочитали и согласны с пользовательским соглашением http://www . mathnet.ru/rus/agreement

Параметры загрузки:

IP: 54.80 .73 .141

26 апреля 2023 г., 15:46:46

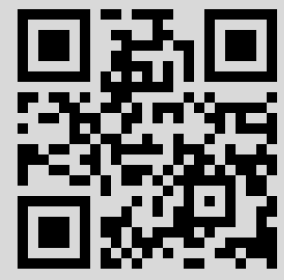




\section{Вычисление двух пар Белого степени 8}

\section{В. А. Дремов}

1. Определения. Пусть $X$ - полная гладкая неприводимая комплексная алгебраическая кривая. Рациональная функция $\beta: X \rightarrow \mathbb{P}^{1}(\mathbb{C})$ называется функцией Белого, если она имеет не более 3 критических значений. Парой Белого называется пара $(X, \beta)$, состоящая из кривой и функции Белого на ней. Пара Белого называется чистой, если критические значения $\beta$ лежат в множестве $\{0,1, \infty\}$ и все прообразы в $\beta^{-1}(1)$ двукратны (т. е. $\beta-1$ имеет лишь в точности двукратные нули). Степенъю пары Белого будем называть степень функции Белого. Чистой паре Белого $(X, \beta)$ соответствует граф $\Gamma=\beta^{-1}([0,1])$, вложенный в поверхность $X$. Прообразы 0 вершины $\Gamma$, прообразы 1 лежат на ребрах $\Gamma$, количество ребер $\Gamma$ равно $(\operatorname{deg} \beta) / 2$. Топологией своего графа и его вложения в ориентированную поверхность пара Белого определяется однозначно с точностью до изоморфизма [1]. Далее 2-вычетом мероморфного квадратичного дифференциала $\Omega$ в точке $P$ называется коэффициент $\operatorname{Qres}_{P}[\Omega]=c_{-2}$ при мономе $x^{-2}(d x)^{2}$ в разложении в ряд $\Omega=\sum_{k} c_{k} x^{k} d x^{2}$ по локальной координате $x$, для которой $x(P)=0$.

2. Введение. Если чистая пара Белого имеет степень 8 , то соответствующая кривая имеет род не более 2. В настоящее время существует полный список [2] чистых пар Белого степени не выше 8. Случай четырех различных пар Белого рода 2 был исследован в работе [3]. Вычисления для рода 0 проведены стандартными средствами. Для рода 1 были разработаны специальные методы. Метод, использованный для двух графов, изображенных на рис. 1, описан в данной статье.

(a)

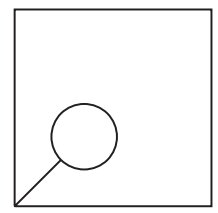

(b)

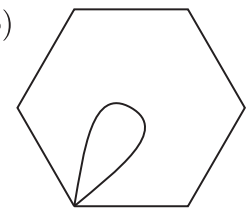

Рис. 1. В каждом из изображающих рисунки многоугольников противоположные стороны попарно склеены

3. Схема вычислений. Составляем систему уравнений для квадратичных дифференциалов вида $\mathrm{MP}(f)=\frac{1}{4 \pi^{2}} \frac{d f^{2}}{f(1-f)}$. Из системы находим квадратичные дифференциалы $\operatorname{MP}(\beta)$ и $\operatorname{MP}\left(\beta^{-1}\right)$, после чего искомая функция $\beta$ может быть найдена из тождества $\beta \mathrm{MP}\left(\beta^{-1}\right)=-\mathrm{MP}(\beta)$. Квадратичные дифференциалы вида $\mathrm{MP}(f)$ рассматривались и ранее (см., например, [4]). Они тесно связаны с пространством модулей кривых. Эта связь выходит за рамки данных рассмотрений, поэтому упомянем о ней лишь кратко.

Отступление. Дифференциалы, построенные по рисункам, естественно соответствуют центрам клеток одного из стандартных разбиений оснащенного пространства модулей. А именно, существует конструкция, позволяющая по рисунку $D$ и положительным действительным числам на его ребрах построить кривую $X_{1}$ и квадратичный дифференциал $\Omega$ на этой кривой. В частном случае, когда все числа равны 1 , мы получим по этой конструкции пару $\left(X_{1}, \Omega\right)$, соответствующую паре Белого $\left(X_{2}, \beta\right)$ следующим образом: $X_{2}=\phi\left(X_{1}\right), \Omega=\mathrm{MP}(\beta(\phi))$, где $\phi$ - изоморфизм. 
Мы рассматриваем следующую систему уравнений на квадратичные дифференциалы $g, h$ и константы пропорциональности $c_{0}, c_{1}, c_{2}$ :

$$
\begin{array}{ll}
\quad \beta=c_{0} g / h ; & \\
(g)=3 A_{1}+A_{2}-2 C_{1}-2 C_{2}, & g=c_{1} \Omega(\beta) ; \\
(h)=-2 A_{1}-2 A_{2}+5 C_{1}-C_{2}, & h=c_{2} \Omega\left(\beta^{-1}\right) ; \\
\operatorname{Qres}_{C_{1}}[g]=49 \operatorname{Qres}_{C_{2}}[g], & 9 \operatorname{Qres}_{A_{1}}[h]=25 \operatorname{Qres}_{A_{2}}[h] .
\end{array}
$$

Ответ получаем с коэффициентами из поля степени 4 над $\mathbb{Q}$, но, сделав бирациональную замену координат, можно перейти к полю $\mathbb{Q}[\sqrt{105}]$. Численно изобразив вид рисунка по паре Белого, мы определяем соответствие между топологией рисунков и парами Белого на плоских комплексных кривых. Получаем следующий результат.

4. Основной результат. Рассмотрим кривую $X$ рода 1 , уравнение аффинной части которой имеет вид

$$
y^{2}=f(x),
$$

где $f$ - многочлен степени 3. Функцию $\beta$ на ней запишем в виде

$$
\beta=\frac{n_{0}-n_{1}+1}{2}+y \sqrt{\frac{\left(n_{0}-n_{1}\right)^{2}-2\left(n_{0}+n_{1}\right)+1}{4 f}}
$$

(в терминах $\beta=P(x)+y Q(x)$ можно записать $\left.n_{0}=P^{2}-f Q^{2}, n_{1}=(P-1)^{2}-f Q^{2}\right)$.

Введем для упрощения формул обозначение $\gamma= \pm 45 \sqrt{105}$. Тогда

$$
\begin{aligned}
n_{0}(x) & =\frac{7^{3}(39 \gamma+17983)}{2^{9} \cdot 3^{5} \cdot 5^{3}} \frac{(x+5)^{3}(x-3)^{5}}{64 x-105+\gamma}, \\
n_{1}(x) & =\frac{7^{3}(39 \gamma+17983)}{2^{9} \cdot 3^{5} \cdot 5^{3}} \frac{\left(x^{4}-30 x^{2}+40 x+(135 \gamma-60825) / 14\right)^{2}}{64 x-105+\gamma}, \\
f(x) & =420 x^{3}-(119+9 \gamma) x^{2}+14(1515-\gamma) x+420(420-\gamma) .
\end{aligned}
$$

В этих обозначениях формулируется результат:

Теорема. Рисунку 1 (а) соответствуют положительное значение

$$
\gamma=+45 \sqrt{105}>0
$$

значение $j$-инварианта, челая часть которого равна 1315 , и пара Белого $\left(y^{2}=f(x)\right.$, $\beta(x, y))$, определенная выше.

Рисунку 1(b) соответствуют отрицательное значение

$$
\gamma=-45 \sqrt{105}<0
$$

значение $j$-инварианта, целая часть которого равна 20, и пара Белого $\left(y^{2}=f(x)\right.$, $\beta(x, y))$, определенная выше.

\section{Список литературы}

[1] S. K. Lando, A. K. Zvonkin, Low-dimensional topology, II, Encyclopaedia Math. Sci., 141, Springer-Verlag, Berlin, 2004. [2] Н. М. Адрианов, Н. Я. Амбург, В. А. Дремов, Ю. Ю. Кочетков, Е. М. Крейнес, Ю. А. Левицкая, В. Ф. Насретдинова, Г. Б. Шабат, Фундамент. и прикл. матем., 13:6 (2007), 35-112. [3] Н. М. Адрианов, Г. Б. Шабат, УМН, 60:6 (2005), 229-230; англ. пер.: N. M. Adrianov, G. B. Shabat, Russian Math. Surveys, 60:6 (2005), 1237-1239. [4] M. Mulase, M. Penkava, Asian J. Math., 2:4 (1998), 875-920.

\section{В. А. Дремов (V. A. Dremov)}

Московский государственный университет им. М. В. Ломоносова

E-mail: dremov@mccme.ru
Представлено А. В. Михалёвым Принято редколлегией 07.12 .2008 